\title{
Les Rayons cathodiques dans le champ magnétique
}

\author{
Par P. VILLARD, \\ Docteur is sciences. \\ Professeur suppléant au Conservatoire National des Arts et Metiers.
}

A la suite d'observations direrses, d'anomalies singulières, signalées par divers auteurs à propos des aspects présentés par les ampoules cathodiques placées dans le champ magnélique, je me suis proposé de reprendre les expériences de Hittorff sur l'enroulement des rayons dans un champ, ainsi que celles, plus anciennes, de Plücker.

Un rajon cathodique est, comme on sait, la trajectoire d'une particule électrisće, dont la charge, toujours négative, est celle de 1 atome d'hydrogène électrolytique $\left(7 \times 10^{-10}\right.$ unités électrostatiques ou $2,5 \times 10^{-19}$ coulombs), mais d'une masse environ deux mille fois moindre.

Ces corpuscules, comme on les appelle, ne peuvent guère ètre étudiés que dans des ampoules où l'on a réalisé un vide très avancé pour supprimer tout obstacle au mouvement, et avec des dillérences de potentiel de quelques milliers de volts au moins et souvent supéricures à 25000 volts. Exceptionnellement, on peut descendre à 500 volts. Les vitesses observées dans ces con litions' ${ }^{1}$ sont énormes; elles se chiffrent par milliers de kilomètres à la seconde, el peuvent dépasser le tiers de la vitesse de la lumière.

La masse de ces corpuscules pourrait ètre matérielle au sens ordinaire du mol (hydrogìne par exemple); certains auteurs pensent que les propriétés de l'électricité suffisent à expliquer l'inertie de ces corpuscules et les considèrent comme des atomes d'électricité libre (électrons).

L'apparence de masse serait un phénomène purement électro-magnétique. Si, en effet, on déplace une charge électrique, il en résulte un courant de convection qui produit autour de lui un champ magnétique. L'accélération qu'il a fallu appliquer à cette charge

1. La vitesse est proportionnelle à la racine carrée de la chute de potentiel motrice. En effet, soient $\boldsymbol{m}$ la masse, $e$ la charge, $u$ la ehute de potentiel, $v$ la vitesse.

On a évidemment

$$
v=\sqrt{\frac{\mathrm{U} \frac{2 e}{m}}{2}}^{\frac{1}{2} m v^{2}=e \mathrm{~L},} \text { (unités C. G. S.), }
$$

$\frac{e}{m}$ est voisin de $1,8 \times 10^{\overline{ }}$. U est le nombre de volts multiplie par $10^{8}$. Pour 1000 volts, on aura

$$
\begin{aligned}
v & =\sqrt{1000 \times 10^{8} \times 5,6 \times 10^{7}} \\
& =\sqrt{3,6} \times 10_{9} \frac{\mathrm{cm}}{\mathrm{sec}}=1,7 \times 10^{4} \frac{\mathrm{km}}{\mathrm{sec}},
\end{aligned}
$$

soit $17000 \mathrm{~km}$ par seconde.

Pour 100090 volts ce serait dix fois plus, soit $170000 \mathrm{kms}$. T. III. pour lui donner une vitesse se traduisant ainsi par la production d'un champ magnétique qui emmagasine de l'énergie, un travail a dù ètre dépensé pour produire la vitesse en question : cést bien là le caractère de l'inertic; si maintenant nous voulons arrèter cette charge en mouvement, détruire sa vilesse, cet arrèt restituera l'énergie emmagasinée, exactement comme l'arrèt d'un corps en mouvement ne peut se laire qu'en absorbant sa force vive, en la transtormant par exemple en chalcur.

Supposons au contraire l'alome électrique lancé et se mourant uniformément en ligne droite : le courant ćlectrique qu'il représente est constant, le champ produit ne varie pas, aucune dépense de trarail n'est done nécessaire; c'est identiquement ce qui arrive pour un objet matéricl en mouvement uniforme.

Ainsi l'atome de fluide électrique ou électron, par cela môme qu'il est une charge, possède une inertic, il a une réritable masse, mais celte masse est d'une singulière nature, bien faite pour dérouter la notion d'invariabilité, de constance, yue nous attachons ì l'expression : masse d'un corps. Considérée en effet comme un phénomène électromagnétique, la masse augmente avec la vitesse; cet accroissement est sans doute faible aux vitesses courantes (projectiles, et mème planèles ou comètes), mais il devient très sensible quand on arrive au tiers ou à la moitié de la vitesse de la lumière (ritesses cathodiques) : pour la vitesse même de la lumière la masse serait inlinie.

Cette notion nouvelle de la nature purement électrique des corpuscules conduit d'ailleurs à cette conséquence paradoxale qu'clle n'exclut nullement la présence de matière ordinaire dans ces mèmes corpuscules; il est en eflel naturel, puisque l'électromagnitisme explique l'inertie, d'admettre que toule inertic, partant toute masse, est de nature électrique. La matière, telle que nous la concerons, serait un assemblage d'électrons, son inertie serait de la self-induction et la masse d'un objet varierait avec la vilesse jusqu'à devenir infinie pour $500000 \mathrm{~km}$ : s. C'ést dire qu'un projectile ne pourra jamais ètre lancé asec la vitesse de la lumière.

$\mathrm{S}$ il en est ainsi, rien ne s'oppose à admettre que les corpuscules cathodiques contiennent par exemple de l'hỵdrogène, puisque cela n'empeche en rien leur masse d'ètre d'origine purement électromagnétique.

Quelle que soit d'ailleurs l'hypothèse admise, le ravon cathodique est la trajectoire d'une masse élec- 
trisée. Il est équivalent ì un courant el. comme tel, sensible à un champ magnétique.

Champ uniforme. - le calcul de la modilication qu un champ uniforme lera subir à la trajectoire est un problème de balistique très simple : supposons le corpuscule lancé perpendiculairement aux lignes de force. Il est soumis à une force constamment perpendiculaire à sa vitesse; celle-ci reste donc invariable. La direction seule est modifiée, tout en restant évidemment dans un plan perpendiculaire au champ (plan contenant la vitesse et la force). La trajectoire est donc plane. D’autre part la force est constante, l'accélération l'est donc égalenent et la ligne plane suivic par le projectile cathodique devant rester identique à ellemime sur tout son trajet, ne peut ètre qu'une circonférence parcourue d'un mourement uniforme.

$\mathrm{Si}$ le rayon incident est oblique au champ, sa vitesse peut être résolue en deux composantes : l'une, perpendiculaire au champ, donne une circonférence parcourue à vitesse constante (arc proportionnel au temps); l'autre, parallèle au champ, produit un déplacement du mobile proportionnel aussi au temps et non modifié par le champ. Nous avons ainsi un point dont l'ordonnéc est proportionnelle au temps comme son abscisse curviligne : ce point se déplace donc sur une hélice dont le diamètre est celui de la circonfërence correspondant à la composante de la vitesse perpendiculaire au champ.

Le rayon de cette circonférence, ou du cylindre sur lequel s"enroule l'hélice, est évidemment en raison inverse du champ et proportionnel à la vitesse; il est donné par la formule très simple

$$
\mathrm{R}==\frac{m}{e} \frac{v}{\mathfrak{H}} \text { (unités électromagnétiques C. G. S.), }
$$

$\frac{m}{e}$ étant le rapport de la masse à la charge, $v$ la vitesse ou sa composante perpendiculaire au champ, $\mathfrak{H}$ le champ; $m$ et $e$ n'interviennent évidemment que par leur rapport, car, si l'on rend solidaires par exemple deux projectiles identiques, ce qui double charge el masse, la trajectoire du système reste celle de chaque projectile.

Supposons maintenant yu'un rayon cathodique soit émis par un point d'une cathode. Ce point d'émission liest géométriquement qu'un point de la trajectoire; il se trouve donc, si le rayon est perpendiculaire au champ, sur la circonférence. Autrement dit, tous les rayous émis par un point dans un plan perpendiculitire au champ, quelles que soient leurs vitesses, décriront des circonférences contenant le point d'émission et, par suite, reviendront tous à ce point. Si les vilesses seules diffèrent, mais non les directions, on olstiendra une famille de circonférences toutes tangentes à la direction initiale au point d'émission, et de diamètres différents (fig. 1).
Pour un obsemalemer regiandiunt diuns le sems du champ, l'enroulement esl diecril diuns le sens des aiguilles d'une montre.

En considérant la lormule précédente on voil qüil revient an mème de fiare varier le champ ou la vitesse;
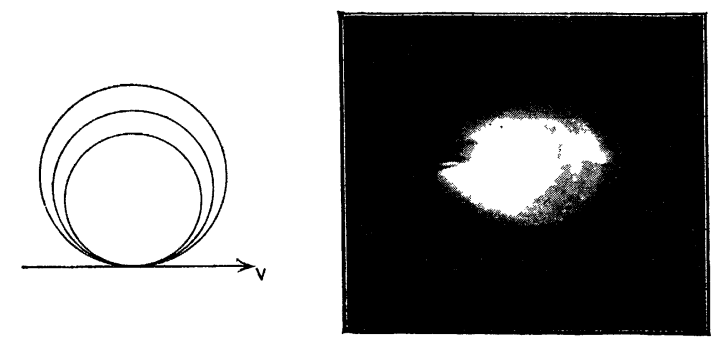

Fig. 1 et 1 bis. - Enroulement de rayons de viless's dillerentes et de mème direction initiale trois rayous seulement figurés'.

arec des rayons de vitesse unique on obtiendrait, en faisant varier le champ, l'aspect des figures 1 et 1 bis. Le diamètre de chaque circonférence serait en raison inverse du champ.

Si les rayons sont obliques au champ, et de mème direction, on aura une famille d'hélices dont le pas et le diamètre sont l'un et l'autre proportionnels à la vitesse et dont le rapport est celui des deux composantes de cette vitesse, c'est-à-dire constant. Ces hélices seront donc semblables et cnroulées sur des cylindres parallèles au champ; de mème que les circonférences précédentes passaient toutes par le point d'émission, lescylindres dont il sagit auront tous une génératrice commune passant par ce mı̀me point (fig. 2).

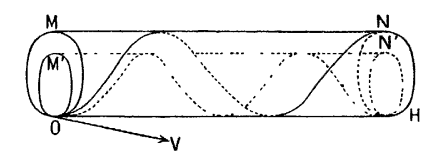

Enfin, si les directions et les vitesses sont quelcon-

Fig. 2. - Enroulement hélicoïdal de deux rayons de mème direction et de vitesses différentes dans un cham! uniforme (hélices semblahles).

ques, on aura des hélices quelconques, mais les cylindres d'enroulement conserveront la génératrice commune dont il s'agit.

Pour réaliser des conditions roisines de ces cas théoriques, on prend une cathode plane ou faiblement concare, de 8 millimètres à 12 millimètres de dia-

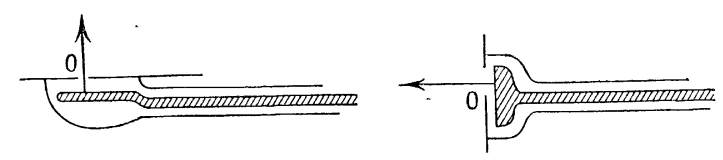

Fig. 3. - Cathodes employées pour oblenir un faisceau cathodique fin.

mètre, enfermée dans une coupe en verre sur les bords de laquelle s'applique une lame de mica percée d'une ouverture de $\mathbf{5}$ millimètres à 4 millimètres de diamètre (fig. 5). L'émission se fait uniquement par le point de la cathode situri en face du centre de cette ou- 
verture, yu elle soit près du milieu ou des bords de la cathode; celle-ci étant assez large, le champ électrique est sensiblement uniforıne dans son voisinage, seule région de l'ampoule où il soit intense, et l'on a un faisceau presque parallèle et très étroit, sulfisant pour observer dans de bonnes conditions Jes circonférences d'enroulcment et quelques spires d'hélice. Avec une cathode enferméc dans une coupe très aplatie, on peut très facilement observer le retour des rayons all point d'émission.

Quelques précautions sont à prendre pour l'usage de ces appareils fragiles. Les décharges irrégulières de la boline de Ruhmkorff conviennent mal : il faut employer soit la machine statique, soit un Iransformatcur is haut voltage qui fournit un courant d'une parfaite régularité. On met un rhéostat sur le primaire et une résistance liquide sur le circuit secondaire.

Le vide doit ètre fait sur l'oxygène pur, dans lequel les rayons cathodiques sont extrèmement visibles. Cet

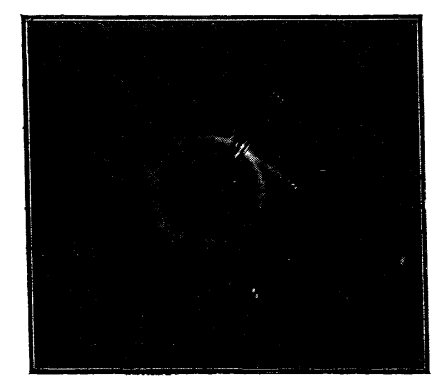

Fig. 4. oxygène s̈épuisant assez vite, on le renou clle en chauffant un petit tube fixé à l'ampoule et contenant un peu doxyde d'argent pur, préparé sans faire usage de filtres et lavé sculement par décantation.

La figure 4 représente un enroulement photographié dans une ampoule de ce geure. Sur la figure 5 on a photographié le faisceau initial et son enroulement dans une série de champs de diverses ra-

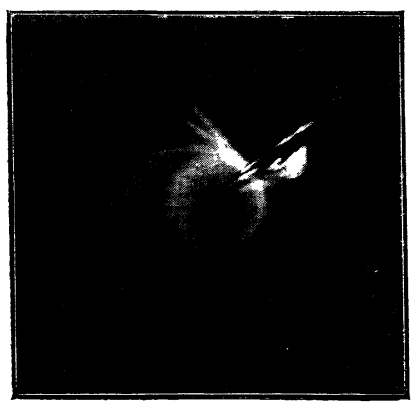

Fig. 5. - Photographic diun faisceau calhodique et enroulement de ce faisceau dans des champs successifs de plus en plus intenses.

leurs, jusqu'ì 1200 unités environ. On reconnait l'aspect prévu par la figure 1 .

Pour étudier les hélices il faut diaphragmer le faisceau de manière à éliminer tous les ravons sensiblement écartés de la direction principale. On $\mathrm{y}$ arrive aisément en plaçant. en avant de la cathode, un diaphragme de mica percé $d$ une trìs petite ouverture. Celle-ci pourra ìtre sur la ligne de force passant par le point d'émission (ligne OH de la figure 2). Ln révglant convenablement l'in-

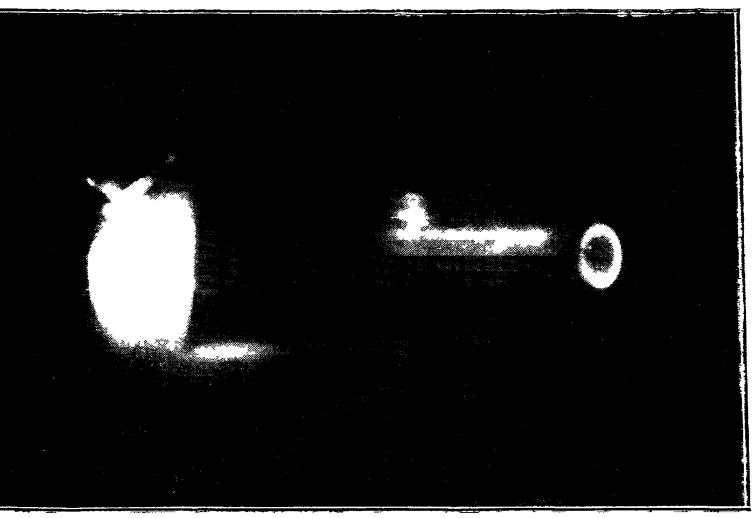

Fig. 6. - Ilèice cathodique dans un champ unforme. On voit que le faisceau est plus ètroit que l'ouverture d'émission 'cathode de la fig. 3). Toutes les spires passent par la ligne de force issue du point d'émission. (Ce faisceau est diaphragmé aprés une spire).

clinaison de la cathode (perpendiculairement ì (01) et la distance du diaphragme on épure le faisceau après une ou deux ou trois spires complètes; on reste d'ailleurs maitre de faire varier à volonté le diamètre des cylindres d'enroulement puisqu' on diaphragme sur la génératrice communc à tous.

Il est souvent préférable de diaphragmer au contraire sur une ligne telle que $\mathbf{I N}$ ou $\mathbf{I I}^{\prime} \mathbf{Y}^{\prime}$, parce que les rayons de vitesses différentes sont alors plus séparés. Mais le diamètre des hélices est alors imposé, et c'est en réglant la valeur du champ qu'on arrive ì faire passer les rayons par ie trou ménagé diuns lia lame de mica.

Ce mode d'épuration du faisceau, après un nombre convenable de spires ou de demi-spires, donne beaucoup de précision aux observations et permet d'observer juscu'à dix spires complètes.

La figure 6 représente des enroulements aiusi obtenus. On voit que les choses se passent tout ì fait conformément à la théorie.

Ghamp non uniforme. - Les rayons s'enroulent encore suivant des courbes qui rappellent l'hélice, mais cet enroulement ne se fait pas sur un tube de force.

Le problème ne peut ètre abordé géométriquement que dans des cas très simples : supposons, par exemple, un champ tel qu'on l'obtient entre les pòles de deu. électro-aimants droits placés dans le prolongement l'un de l'autre. Ce champ est de révolution, il possède un plan équatorial; dans ce plan et dans des plans parallèles il décroit de l'axe à la périphérie (champ en forme de barillet).

Considérons un rayon lancé dans le plan équatorial suivant une direction $\mathrm{Al}$ l passant par l'axe 0 du champ (fig. 7) et donnons-nous la position des lignes d'égale force magnétique (circulaires) et la valeur de lit force sur chacune de ces lignes (déeroissante de l'ase ì lia 
péripherie, celledécroissance élant représentéc par un écartement progressil des lignes d'ígale force).

Si le champ était uniforme, comme il est perpendiculaire au plan de la figure, le rayon s'enroulerait suivant we certaine circonfírence do centre P, indiquée en pointillé. Mais, à mesure qu'il s’éloigne de la

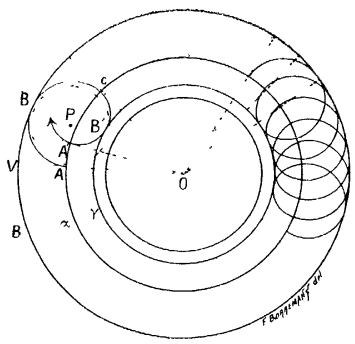

Fig. T. - Construction de la spirale cathodique à double enroulement dans le plan equatorial d'un champ non uniforme produit entre deus pòles magnétiques. ligne $\alpha$, la lorce diminue, le rayon de courbure de la trajectoire augmente et celle-ci suécarte de la circonférence. Arrivé sur une certaine ligne $\beta$, en $B$, le projectile cathodique est dirigé tangentiellement ì $\beta$ et, continuant à tourner, il rencontre dans le sens croissant les champs qu'il avail, de 1 à $B$, traversés dans le sens décroissant. Le problème est analogue à celui du mirage, et l'arc décrit, BC, sera symétrique de IB par rapport à OB. Au point $\mathrm{C}$ le rayon sera dirigé suivant $\mathrm{CO}$ et la courbure en ce point sera la mème qu'en $A$. Il est essentiel de remarquer que le point $G$ ne peut ètre sur la circonférence $\mathrm{P}$, et qu'il est en dehors, comme le montre la figure. Si, en effet, on avait deux ares $\mathrm{IB}$, BC:, symétriques, tels que B et $C$ soient sur la circonférence P, la courbure passerait en B par un maximum, tandis qu'elle y passe par un minimum. $C^{\prime}$ est done en dehor's de la circonférence.

I partir de C le rajon rencontre des champs plus intenses que le long de la ligne $\nsim$ et croissants; la courbure dela trajectoire aug-

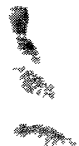
mente jusqu'à un certain point $B^{\prime}$ où elle est maxima, puis décroìt à mesure que le ravon revient vers la ligne $\alpha$, qu'il atteint en $\mathrm{A}^{\prime}$; les deux $\operatorname{arcs} \mathrm{CB}^{\prime}, \mathrm{B}^{\prime} \mathrm{A}^{\prime}$ sont symétriques et au point $\mathrm{A}^{\prime}$ le rayon se retrouve dans les conditions initiales. Mais il est décalé de la quantité $A A^{\prime}$. Comme il n'y a aucun obstacle en ce point, il recommence une deuxième spire identique à la précédente, puis une troisième el ainsi de suite indéfiniment; c cost ce qui est représenté sur la même ligure à droite.

linsi, tandis que dans un champ uniforme le pro-

ig 8. - Spirale cathodique à double enroulement conforme at tracé de la fig. 7. sence dobstactes recommencerail indélinimenl lis mime circontérence, il décrit ici une spirale particuliope lelle qu'après chaque tour complet le rayon est décalé d'une quantité constante; autrement dit, la spirale ne se referme pas sur elle-mème; elle constitue un double enroulement : $1^{\circ}$ autour du champ magnétique; 2" autour de l'axe de ce champ.

Iu lieu de considérer un rayon partant d'un point tel que A dans une direction $0 \%$, on aurait pu le prendre dirigé d'une manière quelconque. Le point d'émission n'élant pas autre chose qu'un point de la trajectoire, cela reviendrait à commencer la spirale précédente par un quelconque de ses points.

Considérons maintenant le cas beaucoup plus intéressant d'un rajon lancé obliquement dans un champ scmblable au précédent.

Supposons, pour simplifier, li calhode dans le plan équatorial et le rayon lancé obliquement à ce plan, du còté d'un des pôles, par conséquent. Si le champ était unilorme, apris un tour complet le rayon rencontrerait de nouveau la ligne de force passant par le point d'émission. Mais, comme dans le cas précédent, le champ est plus intense près de l'axe que loin de cet axe. L'are d'hélice extéricur s’allongera done comme le laisait la courbe $\mathrm{ABC}$ de la figure 7 par rapport à la circonférence, puis deviendra plus serré comme l'are $\mathrm{CB}^{\prime} \mathrm{A}^{\prime}$ et, de mème que le rajon ne repassait par par A, il ne reviendıa pas ì la ligne de force initiale, il y aura un décalage : à chayue spire ce décalage se reproduira, et le rayon s'écartera de plus en plus de la ligne de force en yuestion.

En mème temps ce rayon,-avançant vers un pòle, rencontrera des champs de plus en plus intenses. Assimilons ce champ, sur une petite étenduc, au champ divergent à lignes de force rectilignes, produit par un pòle isolé. M. Poincaré a démøntré que dans ce cas le rayon s'enroule suivant une ligne géodésique situće sur un còne; or une semblable ligne, après s'ètre rapprochée du sommet du còne, rebrousse chemin et s'en éloigre indéfiniment. Autrement dit, le pas de l'hélice diminue plus vite que son diamètre, devient nul sans que celui-ci le soit, puis devient négatif; le corpuscule s'éloigne alors du pòle et le diamètre des spires recommence à croìtre.

Nous pouvons donc sinon démontrer, du moins admettre, qu'il y aura à la fois le phénomène du rebroussement de la trajectoire hélicoïdale de pas variable, et en même temps le phénomène de décalage déji décrit, le premier dù à la convergence du champ vers le pôle, le second à la plus grande intensité de ce champ prìs de son axe.

Le rayon s'enroulera ainsi en spires décroissantes sur une surface fusiforme oblique par rapport ì la ligne de force passant par la cathode. Cet enroulement s'arrètera à une certaine distance du pòle, s'en éloignera ensuite, puis s'arrêtera de nouveau à la même 


\section{\&as Les rayons cathodiques dans le champ magnétique. sos 101}

distance de l'autre pòle, reviendra vers le premier, et ainsi de suite, sans que le rayon puisse rencontrer les parois de l'ampoule si celle-ci contient les pôles ou au moins en est très prìs.

Quant au décalage, il ne dépend que de la condensation du champ vers l'axe et, par suite, a licu tout lo temps dans le même sens, produisant comme pour la figure 7 un cnroulement général centré sur l'ave de électro-aimant, et parcouru dans le sens des aiguilles d'une montre pour un observateur regardant dans la direction du champ.

On se représentera asse\% bien cotte trajectoire en imaginant un long ressort en hélice qu'on reploierait en zzigzag, et qu'on poserail sur un baril de manière à en faire le tour. La figure $\mathbf{9}$ montre un exemple de ce phénomène, on voit l'aller et le relour du rayon

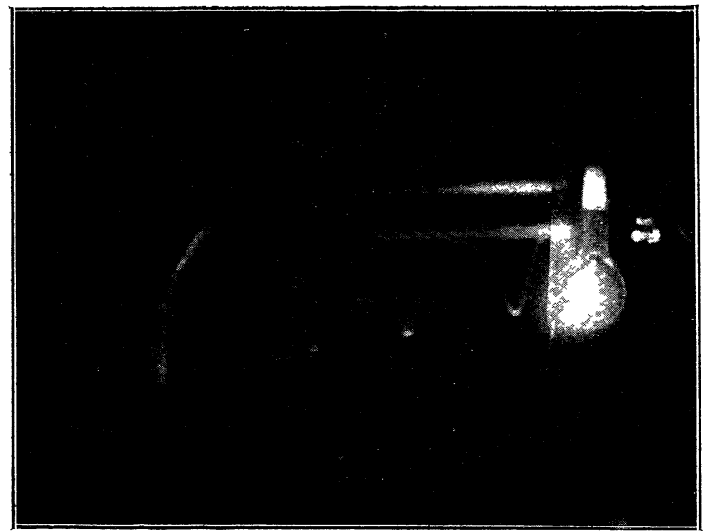

Fig. 9. - Relroussement et décalage d'une spirale cathodique dans un champ non uniforme (grandeur naturelle). On roit en même temps deux faisceaux magnéto-cathodiques.

ainsi que le décalage (une lame de mica arrèle le rayon après le premier aller et retour).

la figure 10 est le calque exact d'une photographie

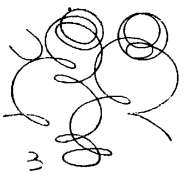

Fig. 10 . de l'enroulement ${ }^{1}$,

Dans une ampoule un peu vaste, on observe aisément un nombre d'aller et de retours suffisant pour couvrir une surface faisant le tour complet de l'axe du champ; l'aspect est celui d'un baril lumineux dont

les doures, sćparées à leurs extrémités, se croiseraient en leurs milieux.

Rayons magnétocathodiques. - Indépendamment de l'enroulement, simple ou componi, des rajons cathodiques, le champ magnćtique fait apparaitre un

1. La reproduction de l'épreuve en photogravure cût íté mauvaise à cause de la faiblesse des spires d'orde liné nécessairement un peu étalées et par suite moins brillantes que les premières. Le dessin au trait peut au contraire itre reproduit sans trame : celui-ci n'est d'ailleurs que le renforcement. à la plume, du positil véritable. phénomène complètement différent, entreru par Plücker puis signalé plus nettement par II. Birholand. et par M. Broca.

Plïcker avait observé que dans un champ magnétique intense la lueur négative d'un tube de Geissler se dispose suivant un tube de forer arant pour base la cathode. M. Birkeland a décrit un phénomène analogue; pour une certaine valeur de la force magnétique à la cathode, celle-ci émet, par toute sa surface, des rayons qui suivent les lignes de force et se terminent à une certaine distance de la cathode sans cause apparente.

En reprenant ces expériences j’ai constaté les faits suivants :

Dans une ampoule où le vide de Crookes est réalisé, si la cathode est plane ou concave, et à peu près adossće à la paroi de l'ampoule, il n'y a émission notable de rayons cathodiques que par le centre de la cathode, du côté libre. Vient-on à placer cette ampoule dans un champ magnétique, les rayons s'enroulent. d'abord en spires d'autant plus serrées que le champ est plus intense, puis, presque brusquement, l'aspect se modific complitement; un large faiscean de rayons apparait, figurant un tube de force qui a la cathode pour base, et les deux faces de la cathode émettent des faisceaux identiques. Les lois ordinaires de l'émission sont complètement transformées.

L'action d'un champ magnélique se manifeste encore d'une autre manière: supposons un tube à rayons $X$, à son vide normal, alimenté par un condensateur isolé.

La décharge passe, abaisse le potenticl du condensatcur, et le tube cesse de fonctionner.

Mais, si on approche un aimant, aussitôt le potenticl explosif est assez abaissé pour que la décharge recommence, pour cesser bientòt, après un nourel abaisse-

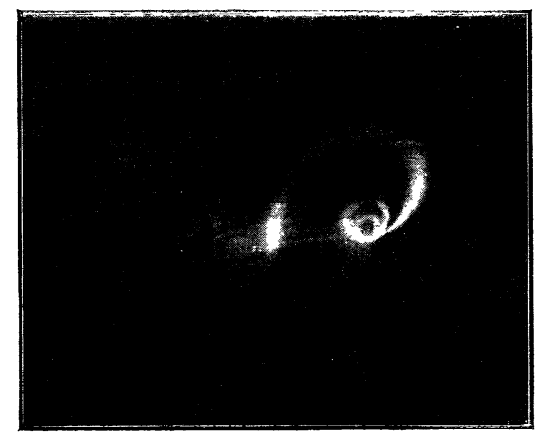

Fig. 11. - Lieu de l'estrémité d'un faisceau cathodique arrité par un plan fluorescent et placé dans un champ croissant.

ment, assez considérable, du voltage du condensateur.

Ainsi le champ magnétique n'a pas seulement une action directrice sur la décharge électrique, il en modifie profondément le régime, abaissant le roltage 
explosif' ol changeant complitement le régime do l'émission.

si maintenant nous prenons une eathode pourvue d'un diaphragne étroil, semblable à celle de la ligure 5 , les rayons élant dirigés oblirpuement dims un champ uniforme, que nous ferons croilre, les rayons senrouleront d'ahord et leur trace sur la paroi opposéc décrira une courbe (fig. 11) dont les boucles décroissantes passeront toutes par un point five, trace de la ligne de force qui passe par le point d'émission. Ce sera un point asymptotique. Mais, après avoir parcouru une dizaine de boucles par exemple, la tache fluorescente cessera de présenter des mouvéments de giration qui indiquent la continuation de l'enroulemint : ello deviendra uniforme et fixe, et son diamètre

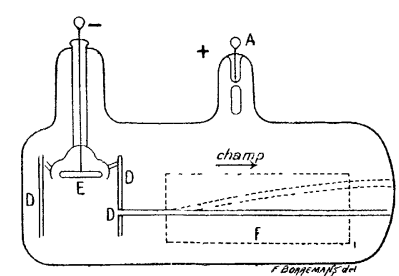

Fig. 12. - Ampoule pour rayons magnctocathodiques. - Dérialion électrustatique de ces rayons. sera cxactement celui du diaphragme catho. dique, l'émission se faisant alors par toute la partie de la cathode et non plus par un seul point.

Ce dispositif peut évidemment, suil y a deux espèces de rayons, donner un mélange; le suivant parait préférable (fig. 12) : une calhode disposée comme le montre la figure est placée entre deux plaques de mica $\mathrm{D}, \mathrm{IJ}^{\prime}$ dont l'une sert à

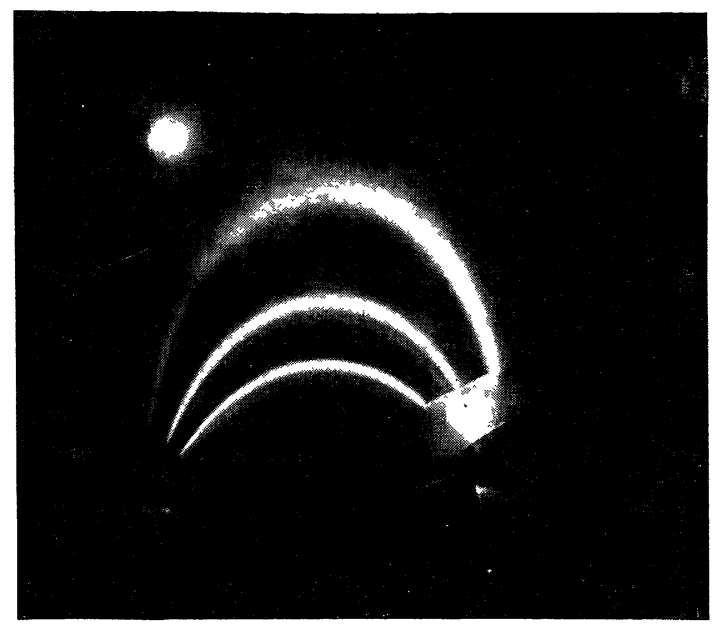

Fig. 15. - Rayons magnélocathediques emis par des ouvertures pratiquées dans une lame de mica placée près d'une cathode, ainsi que par les bords de cette lame. L'ampoule est posée sur un électro-aimant en fer à cheval. las rayons suivent les ligues de force.

protéger le verre de l'ampoule, l'autre étant percée d'une ou deux ourertures telles que 0 .

1. In peut observer une élévation de ce voltage quand las ravons eathodiques sont ramenés par lo champ vers la cathode, paree qu ile électrisent alors los parois do l'ampoule; c'est un etret -reonition.
Pour une valeur convenable de champ lous les rayons émis par lisinroulent sur des cylinders de diamètre inlérieur a la distance EO, aucun ne peul passer par colle ouverture. Mais colle-ci émel un faisecan intense de rayons dirigé suivant les lignes de foree (liz. 15).

si l'on fait passer ce faisceau enter denc platranx patallèles tels que F, en toile mílallique, tris Pilpyprochés, el entre lespuels on rere 111 champ électrostalique, les rayons sont déviés comme le montre la figure 12, parallèlement aux plateaux, c'est-ì-dire que la déviation est perpendicukaire aux lignes de force électrique.

On peut recevoir ces rayons dans un cylindre de Faraday (protégépar une enceinte) : on constate qu ils ne le chargent pas, tandis que les rayons cathodiques ordinaires le chargent négativement.

Pour rappeler le mode de production de ces singuliers rayons, je les ai désignés jar le nom de rayons magnétocathodiques.

II. Fortin a proposi pour ces rayons une théorie qui les ramìnerait à n'ètre que des rayons cathodiques ordinaires de vitesse assez faihle pour que l'enroulement ne soit pas risible.

MI. Fortin a calculé la trajectoire d'un rajon cathodique dans un champ magnétique et un champ électriyue simultanés. Si le rayon est cnroule en spires de très petit diamètre, l'ensemble de l'enroulement est dévié dans un plan presque perpendiculaire à la force électrique, le sens de la déviation variant avec le sens dı champ magnétique et celui de la forcećlectrique.

Or, si les spires sont petites, sil y a un mélange de rayons de diverses vitesses et de directions un peu différentes (c'est toujours le cas), l'ensemble de l'enroulement formera un eỵlindre où l'on ne distinguera rien. et qui simulera un faisceau de rajons rectilignes.

Cette théorie s'applique à un grand nombre de cas, ef il arrive souvent qu'un faisceau d'apparence rectiligne n'est en réalité qu’un faisceau calhodique reconnaissable aux mouvements de giration que présente, quand le champ varie, la lache fluorescente produite sur le verre. La charge d'un cylindre de Faraday est aussi un critérium sùr.

Nos connaissances sont trop incomplètes pour décider si cette théorie très simple et très séduisante ne convient pas à tous les cas. Les phénomènes d'ionisation permettraient peut-ètre d'expliquer l'absence de charge d'un cylindre de Faraday par les rayons magnétocathodiques. Il y a donc lieu, sans faire d'objections proprement dites à cette manière de voir, d'indiquer les anomalies que la découverte de phónomìnes nouveaux pourrait seule faire disparaitre.

En premier lieu, c'est le passage discontinu d'un phénomène à l'autre. Des rayons enroulés en cylindre étroit chargent lien un cylindre de Faraday, puis, brusquement, pour un médiocre accroissement de champ, l'aspect du faisceau change et l'électromètre 
tombe ì réro: le polentiol de décharge n’est pas sensililement modifić, le degré de vide reste identique, il semble bien que c'est la nature des rayons qui n'est plus la mème. En mème temps toute trace d'enroulement ${ }^{1}$, de giration dans la trace du laisceau sur le verre, a disparu.

llans une ampoule où le vide est peu avancé, les rayons magnétocathodiques forment un faisceau qui s'arrite ì peu de distance (quelques centimètres) de son point de départ. Cette distance augmente aver le champ magnétique qui semble ainsi être moteur pour ces rayons. Rien de semblable ne s'observe arec les rayons cathodiques ordinaires.

Si le champ n'est pas uniforme (ampoule posée sur un clectro-aimant en for ì cheral), on a ru que les rayous ealhodiques forment une spirale qui s'arrète ì une certaine distance du pòle, puis rebrousse chemin; mais celte spirale ne suit pas un tube de force, elle forme le zig̨ag déjà décrit. On peut essayer de lancer un rayon cathodifue suivant une ligne de force; il ne la suit que si elle est rectiligne, sinon il ne tarde pas à śen écarter, ce qui est facile à vérifier en se plaçant dans le plan de symélrie passant par les pôles de l'électro-aimant. L'enroulement cathodique s'écarte visihlement de ce plan, le rayou magnétocathodique y reste toujours : il peut s'arrêter plus ou moins loin, suivant le degré de vide et la valeur du champ, jamais il ne rebrousse chemin comme la spirale cathodique.

Rien de tout cela ne constitue cependant une inpossibilité absolue d'admettre la théoric de M. Fortin, parce que nous sommes loin de connaitre toutes les proprićtés des rajons cathodiques et même de savoir si celles que uous connaissons ne sont pas profondément rariables avec la vitesse.

Quoi qu’il en soit, ces rayons magnétocalhodiques présentent avec les rayons cathodiques une remarjuable relation :

Quand un rayon cathodique est placé dans un champ magnélique intense, tous ses points émettent des rayons magnétocathodiques en lesquels il finit par se résoudre complètement.

En voici deux exemples :

Supposons un faisceau cathodique fin enroulé en circonférence; disposons des écrans tels qu'aucune hélice (il y a toujours des rarons non exactement perpendiculaires au champ) ne puisse faire plus d'un demi-tour. Il suffira de prendre pour cela une lame quelconque placée parallìlement aux lignes de force, coupant ainsi longitudinalement tout cylindre d'enrouement. Malgré cela, on voit un cylindre lumineux

1. Avec des faisceaux très fins l'enroulement est tonjours facile à voir parce qu'il remplace le faisceau par le cylindre d'enroulement. Avec des rayons produits par 30000 volts, dans un champ de 500 unités, de diamètre plus grand. le diamètre de le cylindre est voisin de $2 \mathrm{~cm}$. Pour qu'il descendìt à $2 \mathrm{~mm}$, il faudrait que la vitesse fiut dic fois moindre, ot le potentiel de décharge cent fois plus pelit, soit 500 volts. ayant pour hase la circonférence cathodique et figurant un tube de force; ce crlindre niest pas constitué par des hélices, mais par des génératrices magnétocalhodiques.

Formons une hélice calhodique bien netle, de pas défini par un diaphragme à trou placé après les deux premières spires par exemple. Si le champ est faible, cette hélice sera trìs nette, et atteindra la paroi sans affaiblissement appréciable (un rayon cathodique peut facilement atteindre 1 mètre de longueur); si le champ devient intense, l'hélice se resserre en restant semblable à elle-mème, mais sa longueur, de la cathode ì la paroi, reste invariable ${ }^{1}$. Malgré cela, on voit facilement que le rayon cathodique n'atteint plus la paroi : l'hélice, toujours très nette, s'évanouit après huit ou dix spires; le rajon cathodique a disparu, presque complètement, après un trajet inférieur à celui qu'il faisait d’abord.

Cependant le tube de force est toujours visible par fluorescence du gaz, jusqu'ì la paroi. I'hélice s'est résolue en rayons magnétocathodiques et l'on en a la preuve en disposant une lame de mica, mobile à volonté, qu'on amène à couper le tube de force dans le sens de sa longueur; tout ce qui s'enroule est ainsi arrêté. Or, si cette lame est placée à l'origine de l'hélice, elle supprime toute luminescence du gaz après elle ; mais, si elle est placée après la cinquième ou la sixième spire, ou mieux un peu plus loin encore, elle ne supprime presque plus rien, parce qu'il n'y a presque plus de rayons enroulés, mais seulement des rayons magnétocalhodiques qui sont les génératrices du tube de force et qu'une lame parallèle à ces génératrices n'arrète naturellement pas.

L'interprétation est contrôlée par l'observation de la tache fluorescente produite par le bout du faisceau sur le verre. Comme on n'a jamais une hélice géométrique, mais une infinité d'hélices très voisines, cetle tache se compose (dans un champ modéré) d'un are de la courbe représentée figure 10. Cet arc pourra, si l'on veut, former presque un anneau complet. Si l'on fait varier le champ, il se déplace en tournant sur la courbe en question; mais, si l'on coupe l'hélice cathodique en un point quelconque, tout l'arc fluorescent est supprimé.

Si au contraire on a un champ intense, le diaphragme longitudinal qui arrête les hélices ne supprime plus la tache fluorescente : celle-ci, qui est alors un anneau circulaire complet (trace du tube de force), est alors simplement coupée par l'ombre du diaphragme. Quand on fait varier le champ, le diamètre de l'anneau change, mais il reste toujours coupé de la même manière, sans aucune tendance à une rotation quelconque; il est done bien la trace, non d'hèlices

1. Il suffit de considérer le rayon initial comme la diagonale d'un rectangle quion enroule successivement sur des eylindres d'un diamètre de plus en plus faible; l'enroulement occupe toujours la même longueur sur le cylindre, et la longueur de Thélice est aussi toujours la même, étant celle de la diagonale enroulée. 
variables avec le champ mais de rayons rectilignes en lesyucls s'est résolu le rayon calhodique enroulé d'abord en hélice ${ }^{1}$.

Si l'on ajoute à ces singuliers raỵons, varićtí ou non des rayons cathodiques, ceux de M. Goddstein, et les rajons cathodiques non déviables de M. J.-J. Thomson, on voit que nous sommes loin de savoir exactement en quoi consiste une dicharge électrique.

Essai de théorie de l'aurore boréale. II. Arrhénius a émis l'hypothèse que l'aurore boréale est due à des rayons calhodifues émanés du Soleil. C'est là une explication tris plausible de l'origine ou de la cause principale du phénomène, mais cette hypothèse n'explique en rien les caractères de l'aurore ellemême, et dire qu'elle est formée de rayons cathodiques revient sensiblement à considérer l'aurore comme une décharge électrique dans un gaz raréfié, sans donner la raison desparticularités qu'elle présente.

Les particularités en question sont les suivantes:

L'aurore affecte généralement une forme en éventail composé de bandes lumineuses semblables à des faisceaux de projecteurs, convergeant vers le pòle magnétique, mais s'arritant à distance de celui-ci et s'appuyant en quelque sorte sur un are dont l'intírieur est sombre (are auroral).

La base de chaque faisceau sur cet are présente un renforcement notable. La longueur des rayons est variable; souvent on les voit aller d'un pôle à l'autre.

Par instants, des mouvements se produisent dans l'aurore; les rayons se déplacent le long de l'arc auroral comme si l'ensemble tournaii, tantòt dans un sens, tantòt dansl'autre, autour de l'axe magnétique du globe.

Parlois la base des rayons s'élève ou s'abaisse comme si chaque faisceau lumineux était animé d'un mouvement longitudinal (danse des rajons).

Il faul ajouter à cela les taches lumineuses dans le (icl (playues aurorales).

Les phénomènes d'enroulement des rayons cathodiques dans un champ non uniforme permettent d'expliquer lit plupart de ces aspects et mème de discuter l’origine de l'énergie électrique du météore.

Si l'aurore est produite par des particules cathodiques émanant du Soleil ces particules ne peuvent arriver à la Terre que si le champ magnétique solaire est négligeable. En effel, ce champ ćtant de révolution, les rayons cathodiques y produisent l'enroulement composé en zigzag formant autour du Soleil une nappe de révolution dont l's points d'émission feront partie, et qui, par suite, ne pourra s'écarter du Soleil à une distance supéricure à celle de ces points.

Le diamètre des spires d'enroulement scra lui-même

1. Il n'y a pas allongement progressif du pas de l'hélice se transformant en ligue droite, car on la voit s'évanouir sans changer de pas : d'autre part, le diaphragme longitudinal arrêterait l'hélice mìme si elle tendait à se rapprocher progressivement d'une génératrire du cylindre, négligeable devant le diamìtre du Soleil (dans un champ de 1/10 d'unite el pour des particules ayant la vitesse de la lumiere, a diamitre serail de (ion) mitres environ).

Ainsi le champ magnélique solairc est incompatille avec l'arrivéc des particules calhodigues sur lit Terre; Thypothèse d'Arrhénius nous oblige à admettre que ce champ est sensiblement nul, ce qui est possible ru la température élevée de l'astre.

Supposons donc les particules eathodiques entrant dans le champ magnétique terrestre qui s'élend jusqu'au soleil et an dela : leurs trajectoires s'enrouleront, donneront la nappe en zigzag ayant pour axe l'axe magnétique du globe et passant par le licu d'émission, soit le Soleil.

On pourra donc voir peut-c̀tre, très près des pôles, les extrémités de tous les faisceaux d'enroulement, mais comme, à chaque aller et retour, ils devront s'éloigner à une distance égale à celle du soleil, s'ils ne sont pas absorbés en route, ils seront hors de l'atmosphère, dans un vide parfait, et invisibles. Comme il est certain que les aurores horéales ou australes peuvent donner des rayons qui, mème à l'équateur, sont encore dans l'atmosphère terrestre, les raşons cathodiques qui les produisent ne peuvent venir directement du Soleil, à moins de perturbations magnétiques cosmiques modifiant la forme du champ et le trajet des particules pendant leur marche.

On peut peut-être admeltre que ces particules d'origine solairc arrivent assez près des pòles terrestres pour rencontrer des cirrus (qui accompagnent toujours l'aurore).

Ces cirrus peuvent être électrisés négativement parce que les ions négatifs condensent la rapeur d'eau plus facilement que les ions positifs. Or, j’ai observé récemment que les rayons cathodiques à grande vitesse frappant un objet quelconque électrisé négativement provoquent une aboudante émission de rayons par le point frappé, même si, au voisinage de ce point, la chute de potenticl est par elle-mème beaucoup trop faible pour produire spontanément une émission (200 volts suffisent). La vitesse de ces rajons correspond à la chute du potentiel en question. Les cirrus, formés d'aiguilles de glace, constitueraient ainsi de vastes cathodes secondaires. L'émission produite, normale à l'ellipsoïde terrestre, sorait oblique au champ magnétique et l'on serait dans les conditions voulues pour avoir l'enroulement composé déjà décrit.

Rien n'empêche d'admettre l'existence d'autres causes excitatrices d'origine cosmique, radiations ou rayons (on a vu qu'il en est d'insensibles au champ magnélique). Ce qui est essentiel, c’est d'avoir des rayons cathodiques d'origine terrestre.

Nous n'avons ainsi à considérer que l'explication des aspects présentés par l'aurore, celle-ci étant supposée produite par des rayons émis dans notre atmosphère 
Ces rayous sont soumis au champ terrestre qui, malgré sa faible intensité, suffit à donner des enroulements qui n'ont pas 100 mètres de diamètre pour des corpuscules ayant la vitesse de la lumière. Or, le champ terrestre présenle une grande analogie de forme avee celui qu'on oltient enlre deux pôles d'ai mants : les lignes de force seront seulement rentrantes aux pôlcs, e'est-ì-dire plus courbes, ‘'e qui ne peut qu'evagérer les effets observés avec l'électro-aimant.

Considérons donc l'enroulement en zigzag de la figure 10 ; ce sera évidemment celui des ravons dans le champ terrestre dont la direction est généralement oblique au champ ćlectrique de l'atmosphère. Mais, au lieu d'un point, nous aurons une large surface d'émission, un cirrus par exemple. Tous les rayons ainsi émis auront à peu près mème vitesse et mème direction ${ }^{1}$ et donneront des enroulements identiques dont l'ensemble formera un large faisceau dans lequel aucune spire ne sera évidemment visible. Ce faisceau, dont l'épaisseur sera le diamètre d'une spire, et la largeur celle du cirrus plus ce même diamètre, se comportera comme chacun des enroulements dont -il est composé; il se dirigera par exemple vers le Nord, un peu obliquement aux lignes de force, puis robroussera chemin à une certaine distance du pôle pour repartir vers le Sud, la nouvelle direction étant symétrique de la première par rapport à la force magnétique. Vers le pòle Sud, nouveau rebroussement, retour rers le Nord, avec décalage toujours dans le même sens, et, de nouveau, arrêt à la même distance du pôle que précédemment, puis encore départ vers le Sud, et ainsi de suite.

Aux points de rebroussement, qui sont à la même distance d'un pôle si le champ est de révolution, tous les filets cathodiques se replient sur eux-mèmes, et l'expérience de la figure 10 montre que, mème si les directions sont un peu différentes, le reploiement de la spirale ne change pas de position; d'où une condensation de lumière, un renforcement trìs visible résultant du tassement des spires vers le lieu de rebrous-

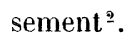

Représentons comme sur un planisphère (fig. 14) la nappe des rayons cathodiques. Le lieu des extrémités des faisceaux fusiformes (ayant comme convergence à peu près celle du champ) étant à une distance constante de chaque pôle (dépendant seulement de la convergence du champ près de ce pòle) sera un parallèle magnétique représenté ici par une droite. Ce sera l'arc auroral avec les renforcements signalés plus haut et observés dans l'aurore boréale.

1. Ils seront, en effet, produits par le champ électrique terrestre.

2. Dans l'expérience de la figure $\mathbf{1 0}$, le faiscean cathodique est devenu presque invisible. par étalement, au bout de dix ou douze spires, el l'on voit cepentant tris bien les boucles terminales correspondant à la ringtième ou vingt-cinquième spire.
La superposition partielle de l'aller et du retour de chaque fuseau (oblique au champ) constituera un faisceau résultant dont la direction movenne sera évidemment celle du chanp. Cette superposition doublant l’intensité lumineuse, ce sera surtout près des ares auroraux que le météore sera visible. Nous avons ainsi

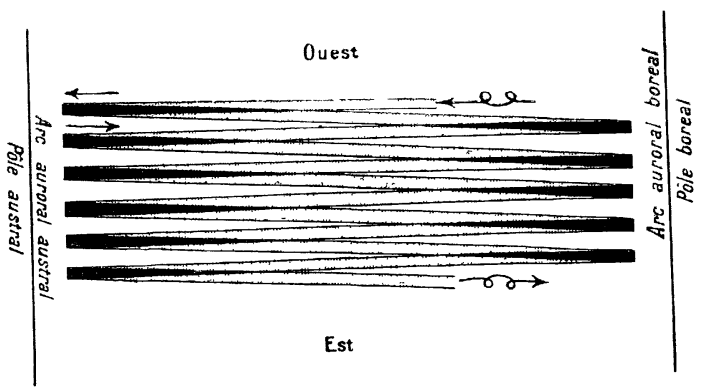

Fig. 14. - Figure schématique (planisphère) de l'enroulement cathodiqu" auroral.

les rayons auroraux disposés en éventail sur l'arc auroral, à l'intérieur duyucl il n'y a rien, puisque les rayons cathodiques n’y peuvent pénétrer.

Si le pas du décalage, c'est-ì-dire la distance des points homologues des spires de rebroussement de deux retours successifs, est supéricur à la largeur du faisceau d'ensemble, les rayons auroraux seront distincts, ce sera l'aurore classique en éventail. Si ce pas est moindre il y aura superposition particlle de deux rayons auroraux voisins; il en résultera des renforcements de lumière simulant les plis d'une draperie (aurore en draperies).

Quel que soit le lieu d'émission, le sens de l'enroulement composé est celui des aiguilles d'une montre pour un observateur regardant le Nord magnétique : c'est le sens de rotation de la Terre Si donc on avance vers l'Est, on rencontrera des fuseaux cathodiques dont le numéro d'ordre sera de plus en plus élevé, et qui, par suite de la déperdition d'énergie sous forme de lumière, seront de plus en plus faibles, jusqu’ì évanouissement complet après une fraction plus ou moins grande du tour de la Terre.

Supposons que l'émission ait lieu sous l'influence d'un rayonnement solaire (II. Buisson a montré que l'ultra-riolet décharge les cristaux de glace électrisés négativement); l'aurore commencera dans l'hémisphère éclairé, et s'avancera vers l'Est en s'affaiblissant; en abordant l'hémisphère obscur elle deviendra visible et le côté de cet hémisphère pour lequel le Soleil vient de se coucher sera évidemment le lieu où l'on verra les fuseaux d'ordre peu élevé, c'est-ì-dire les plus brillants. Ce sera donc aussitôt après le coucher du Soleil qu'on aura le plus de chance de voir une aurore boréale, car, à mesure que la Terre tournera, entrainant l'observateur vers l'Est, il faudra, pour arriver jusqu à lui, des aurores de plus en plus intenses et, partant, plus exceptionnelles. 


\section{6 ags Le Radium. গ⿰氵}

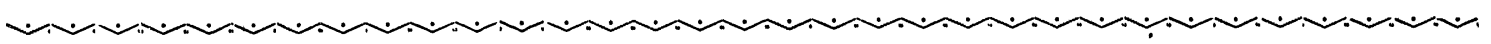

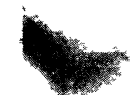
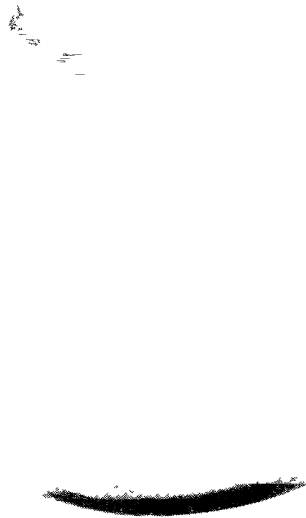

Fig. 15. - Enroulement composé en zigzag dans un champ en barill.t. On voit le renforcement notable de la lummosilé à chaque rebroussement. Ie lieu des divers rebroussements est un pelit rercle vu ici par sa tranche; a l'intérieur de ce petit cerele il u'y a pas de rayons cathodiques.

Supposons maintenant une perturbation magnétique ayant pour effet de changer la convergence du champ

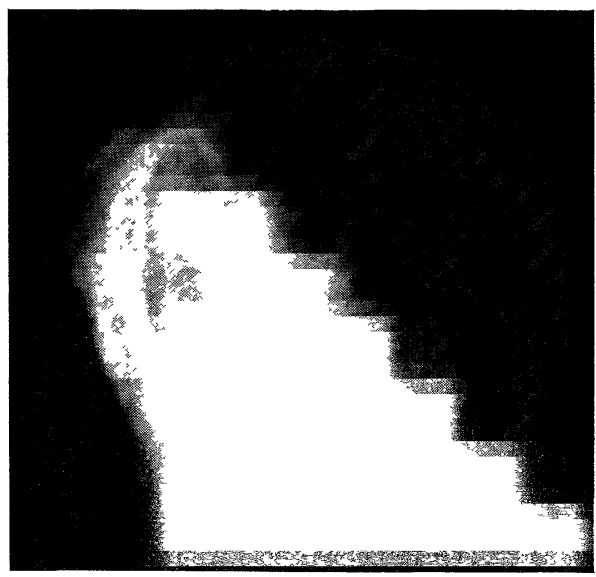

Fig. 16. - Aurore artificiclle. Vue perspective à 45̆ degrés environ de l'axe du champ. (Le diamètre de l'ampoule, 18 centimètres, ètant imposé par les dimensions de l'électroaimant, il a été nécessaire, pour aroir des fuscaux cathodiques relativement sorrés, de donner au champ une valeur élevéc; il en est résulté, surtout près du pòle, une production de rayons magnéto-calhodiques plus forte que dans les deux enroulements précèdents; de la une nettelé beauroup moindre de lare auroral a l'intirieur duquel pénitrent len rayons magnitocathodiques yui peuvent aller jusqu all poile). wrs to poile (ee qu'on réalise expérimentalement on ciloignant ou rapprochant l'un ou l'autre des priter de l'élecetro-aimant). Aussitòt le point de rebroussement du fuseau cathodirfue s'éloignera ou se rapprochera du pôle; on aura le mouvement longitudinal de la danse des rayons.

Si, au contraire, on modific soil la vitesse des rayons (diffírence de potentiel motrice), soit l'intensilé du champ, les spires cathodiques se resserrent par exemple de 10 pour 100 (champ) plus intense ou vitesse moindre), mais, en même temps, les fuseaux se rapprochent; celui qui part du licu d'émission reste à peu près fixe, son diamètre, égal à la largeur du cirrus plus celle d'une spire, change à peine; le second se rapproche du premier de 10 pour 100 ; le troisième se déplace du double et ainsi de suite: on verra done les bases des fuseaur courir le long de l'arc auroral par une vérilable rotation antour de l'axe magnétiyue, rotation dont on se fera une idéc axacte en imaginant que sur la figure 14 on resserre tous les fuseaur à la manière de ces appliques extensibles formées d'entretoises en losanges.

Ce phénomìne est facile à reproduire dans un grand ballon placé entre les pòles d'un électro-aimant, et dans lequel on produit des rayons cathodiques en un point dans une direction convenable. On a la nappe déjà décrite et, si l'on fait varier le champ, cette nappe, sans changer de forme ni de diamètre, s'enroule ou se déroule à volonté autour de son axe, chaque fuseau se déplaçant proportionnellement à son numéro d'ordre. Le phénomène est encore plus saisissant si la source électrique est un alternateur présentant un peu de pompage, d'où résultent des variations périodiques de voltage qui modifient la vitesse cathodıque ct produisent la rotation de l'aurore artificielle. Celte rolation n'est donc qu'un phénomène de pompage naturel du champ terrestre ou du potentiel atmosphérique.

Ainsi, cette étude de l'électromagnétisme cathodique, poussée par l'expérience plus loin peut-être qu'on ne pourrait le faire par l'analyse, conduit non seulement à une explication rraisemblable d'un phénomène naturel remarquable, mais à la possibilité d'en discuter l'origine terrestre ou rosmique'.

1. Ies figures 15,15 et 16 de ce travail nous ont été ohligeamment communiquies par la Socicti Inlernationale des Electriciens. 